\title{
Telepsychotherapy During the COVID-19 Pandemic: The Experience of Lebanese Mental Health Professionals
}

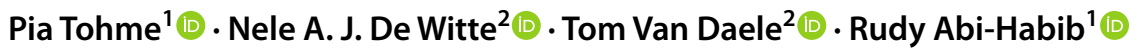 \\ Accepted: 15 April 2021 / Published online: 22 April 2021 \\ (c) The Author(s), under exclusive licence to Springer Science+Business Media, LLC, part of Springer Nature 2021
}

\begin{abstract}
The COVID-19 pandemic has taken the mental health system by surprise, with the state of lockdown forcing businesses to close their doors, including many mental health services. This has driven many psychotherapists and other mental health professionals towards telepsychotherapy, relying on online consultations to provide continuity of care. However, the adoption of telepsychotherapy required major adaptations from both mental health professionals and patients. This study set out to explore the predictors of the use of online consultations and the perceived level of comfort using telepsychotherapy in a sample of 73 Lebanese mental health professionals. Data was collected via online dissemination of a survey. Results show that $82 \%$ of participants made use of online consultations in the past few days, reflecting the adaptation of Lebanese mental health professionals to the atypical newly imposed situation triggered by the COVID-19 pandemic and its consequent lockdown. Having previous experience in the use of online consultations and perceived level of telepresence were significant predictors of the level of comfort of mental health professionals in the execution online consultations. We suggest that more awareness and trainings are required around the practice of telepsychotherapy outside the context of the COVID-19 pandemic.
\end{abstract}

Keywords Lebanon · Telepsychotherapy · Online Consultations · COVID-19 · Mental Health

\section{Introduction}

Online therapeutic services have been around long before the dawn of the COVID-19 pandemic and resulting lockdown. Studies comparing the effectiveness of telepsychotherapy with face-to-face therapy found that both treatment modalities were equally effective in adult populations, with telepsychotherapy even improving treatment adherence for patients suffering from depression and/or anxiety (Kocsis \& Yellowlees, 2018; Poletti et al., 2020). Studies on children and adolescents are scarcer, but nevertheless show promising results in terms of telepsychotherapy effectiveness, in part due to steady technological advancements and the development of new applications (Stewart et al., 2020; Wade et al., 2020).

Rudy Abi-Habib

rudy.abihabib@lau.edu.lb

Pia Tohme

pia.tohme@lau.edu.lb

1 Department of Social Sciences, School of Arts and Sciences, Lebanese American University, Beirut, Lebanon

2 Expertise Unit Psychology, Technology \& Society, Thomas More University of Applied Sciences, Mechelen, Belgium
Unsurprisingly, several challenges and difficulties accompany the use of telepsychotherapy. Early studies looking into the modes of delivery of online psychotherapy, identified strong limitations in the assessment of non-verbal communication and cues regarding gaze direction hampering the therapeutic potential (Ball et al., 1995; O'Malley et al., 1996). However, more recent studies found no compromise in the therapeutic alliance in the online treatment of depression or anxiety, whether delivered by telephone or videoconferencing (Hadjistavropoulos et al., 2017; Jenkins-Guarnieri et al., 2015). On the contrary, some findings revealed that online video consultations enhanced communication as the patienttherapist dyad tended to decrease the pace of interactions in order to pay closer attention to social cues and emotions (Connolly et al., 2020). An important concept in this context seems to be telepresence, which is defined as the perceived online presence or their impression of being physically present (Haddouk, 2015). Telepresence can be experienced to a varying extent by both psychotherapists and their patients and has been known to impact the strength of the therapeutic relationship (Haddouk et al., 2018).

Despite the availability of the necessary technological tools and increasing insights into to processes involved in 
telepsychotherapy, few mental health professionals had fully adopted this practice, or even made the move towards blended psychotherapy combining face-to-face and online consultations before the COVID-19 pandemic (Ryu, 2010). Honing in on the experience of telepsychotherapy during the COVID-19 pandemic, mental health professionals had to transition towards digital psychotherapy in order to provide ongoing care to avoid a potential exacerbation of symptoms of stress and anxiety triggered by the novel coronavirus (Matheson et al., 2020). To best anticipate some of the challenges of telepsychotherapy, including connectivity problems, patient privacy or legal issues, some guidelines are already in place to allow for the best provision of this treatment modality online (Shore et al., 2018; Van Daele et al., 2020). Looking for predictors of mental healthcare professionals' use of telepsychotherapy, some studies focused on psychologists' attitudes towards this delivery method. Findings suggest that more experience in delivering telepsychotherapy is linked to higher confidence in dealing with related challenges. Although this seems to render psychologists more favorable towards this approach, they nevertheless still prefer face-to-face consultations (Connolly et al., 2020; Lindsay et al., 2017). In terms of the effect of therapists' characteristics on the choice of use of online consultations, findings diverge regarding the effects of age, gender and years of professional experience (Glueckauf et al., 2018).

The literature mainly focused on the effectiveness of telepsychotherapy and why some mental health professionals choose to adopt online psychotherapy, whereas others do not. However, little is known regarding the various factors affecting therapists' level of comfort with online consultations. This is especially relevant in the context of the COVID-19 pandemic, where online consultations were an essential tool to provide continuity of care.

The current study therefore looks into reasons why mental health professionals chose to start relying on telepsychotherapy (for instance, being there for their patients or not losing income), as well as the method of delivery of telepsychotherapy (telephone, audio calls via specific applications or videoconferencing) as potential predictors. It furthermore sets out to explore predictors of online consultations in a sample of Lebanese mental health professionals including (1) predictors of the implementation of online consultations, more specifically demographic predictors (i.e., age, gender, years of professional experience) and the influence of having prior experience or previous specific training; (2) predictors of the level of mental health professionals' comfort with online consultations, with a focus on prior experience or specific training in online consultations, level of perceived telepresence and years of experience. Furthermore, the study looks into the reasons why therapists chose to start using this method of consultations (for instance, being there for their patients or not losing income), as well as the method of delivery of telepsychotherapy (telephone, audio calls via specific applications or videoconferencing) as potential predictors.

\section{Material and Methods}

\section{Participants}

73 Lebanese professionals participated ( $\mathrm{n}=56$ were psychologists, $n=4$ were psychiatrists and $n=13$ had other related professions, such as socials workers or speech and language therapists). Participants were recruited through social media, word of mouth, and targeted e-mails. Participants provided online informed consent. They were aged between 24 and 64 years of age $(M=39.09, S D=9.77)$ and $80 \%$ were women.

\section{Survey}

The survey was developed specifically to assess the use of online consultation during the COVID-19 pandemic. The survey was based on the current state of the art technology implementation and the Unified Theory of Acceptance and Use of Technology (Venkatesh et al., 2003). Questions relate to which factors contributed to the use of online consultations in mental healthcare as well as concerns mental health providers might have concerning telepsychotherapy. A combination of open ended questions and multiple choice questions were used in the survey. Perceived level of telepresence ("If you started online consultations recently, how would you qualify your level of telepresence (feeling of being connected with one another) during consultations?") and how comfortable one feels with online consultations ("To what extent do you consider yourself to be comfortable with (the concept of) online consultations?") were scored on a 5-point Likert scale, ranging between 1, very low/highly uncomfortable, to 5, very high/highly comfortable. All data was collected online through the online surveying platform Qualtrics. The survey was designed by the Project Group on eHealth of the European Federation of Psychologists' Associations (EFPA) together with Thomas More University of Applied sciences in Belgium (De Witte et al., 2020). It was, subsequently, translated to 17 languages, including Arabic, by native speakers familiar with the cultural context.

\section{Procedure}

This study is part of a larger international collaboration investigating attitudes towards the use of online consultations during the COVID-19 lockdown, led by the Project Group on eHealth of the European Federation of Psychologists' Associations. Ethical approval was first received from 
the ethical committee of the Department of Applied Psychology of Thomas More University of Applied Sciences (Antwerp, Belgium) and then by the Institutional Review Board of the Lebanese American University in Beirut. Participants could access the survey through a link provided and subsequently had the ability to choose their preferred language of completion. Data was collected between March and May 2020, during the first imposed total lockdown in the country. After reading the information letter and providing online consent, participants proceeded to complete the survey. The survey took approximately $5 \mathrm{~min}$ to complete.

\section{Statistical analysis}

The first aim of this study was to look for predictors of the use of online consultations. First, we explored whether the 3 different user groups (experience with online consultations in the past days, no experience but with intentions to do so, and no intentions at all) differed on these variables, running ANOVAs for age and years of professional experience and Chi Square tests to investigate differences in gender, previous experience using online consultations and having specific training using online consultations (for instance, short online workshops or module part of postgraduate training). We then ran a Multinomial Logistic Regression looking for predictors of the use of online consultations in the significant variables found in order to investigate whether the effect of the variables remained when entered into one model.

Second, we set out to look for predictors of how comfortable telepsychotherapists feel with online consultations. We ran Pearson and point biserial correlations to look for significant associations between the variables, followed by a hierarchical multiple regression looking for predictors of how comfortable one feels with online consultations in variables such as having previous experience or specific training in online consultations, level of perceived telepresence and years of professional experience, as well as age and gender. We ran a multiple linear regression with 6 binary predictors (each presenting one of the options to question 7 relating to why the psychotherapist chose to start using online consultations) and another multiple linear regression with 4 binary predictors (each relating to one of the potential answers to question 10 relating to the method of telepsychotherapy used).

\section{Results}

In our sample, $82 \%$ of participants $(n=60)$ had made use of online consultations in the past few days and $11 \%(n=8)$ planned to start using online consultations. A final $7 \%$ of the sample $(n=5)$ was not interested in using online consultations. On average, participants' years of professional experience ranged between 1 and 40 years $(M=12.78, S D=8.39)$, with $71 \%$ of them having made use of online consultations prior to the COVID-19 pandemic. Only $15 \%$ could rely on prior specific training in providing online consultations. Most frequently indicated reasons for making use of online consultations, noting that participants could select more than one option, related to a need to continue offering care (69\%), to help safeguard public health (59\%) and to meet client demands for online consultations (43\%). Most commonly used technological tools for therapy were video calls (used by $78 \%$ of psychotherapists), followed by chat without video $(25 \%)$ and then email (6\%). Other methods of delivery, such as phone calls or screen sharing, were also implemented $(12 \%)$.

The first aim of this study was to look for predictors of the use of online consultations in demographic variables such as age, gender, years of professional experience, as well as prior experience and previous specific training in online consultations. Analyses of variance revealed that age and years of professional experience were not significantly different across the 3 groups with $F(2,69)=2.09, p=0.13$ for age and $F(2,70)=0.69, p=0.50$ for years of professional experience. Chi-square tests (Table 1 ) revealed that gender, $\chi^{2}=5.72$, $p=0.05$, and previous experience using online consultations, $\chi^{2}=31.39, p<0.001$, were significantly associated with the choice to use online consultations. It was found that more females have used online consultations than males and participants having had previous experience with online consultations were more likely to have used this method of
Table 1 Chi-square tests looking at the association between the use of online consultations and gender, previous experience using online consultations and specific training regarding online consultations

\begin{tabular}{|c|c|c|c|c|c|c|c|}
\hline \multirow[t]{2}{*}{ Use of online consultation } & \multicolumn{2}{|c|}{ Gender } & \multicolumn{2}{|c|}{$\begin{array}{l}\text { Previous experience } \\
\text { using online consul- } \\
\text { tations }\end{array}$} & \multicolumn{2}{|c|}{$\begin{array}{l}\text { Received specific } \\
\text { training in online } \\
\text { consultations }\end{array}$} & \multirow[b]{2}{*}{ Total } \\
\hline & Male & Female & Yes & No & Yes & No & \\
\hline Yes & 11 & 49 & 51 & 9 & 10 & 50 & 60 \\
\hline No, but intend to & 4 & 4 & 1 & 7 & 1 & 7 & 8 \\
\hline No & 0 & 5 & 0 & 5 & 0 & 5 & 5 \\
\hline$X^{2}$ & $5.72 *$ & & 31.3 & & 1.05 & & \\
\hline
\end{tabular}

$* p=0.05 ; * * p<0.001$ 
therapy in the past few days. However, having specific training was not found to significantly affect the current choice for online consultations in our model, $\chi^{2}=1.05, p=0.59$. A Multinomial Logistic Regression, with gender and previous experience using online consultations as predictors, solely revealed the unique effect of previous experience on the use of online consultations, $\chi^{2}=30.69, p<0.001$. Participants with experience using online consultations prior to COVID19 were more likely to have made use of online consultation in recent days.

Second, we set out to look for predictors of how comfortable telepsychotherapists feel with online consultations $(M=3.66, S D=1.24)$ in variables such as having previous experience or specific training in online consultations, level of perceived telepresence and years of professional experience. Pearson correlations showed that the level of telepresence was significantly correlated with how comfortable one feels with online consultations, $r(59)=0.39$, $p<0.01$, but that years of professional experience was not, $r(59)=0.14, p=0.29$. Point biserial correlations showed that having had experience with online consultations prior to COVID-19 was significantly associated with being more comfortable with this type of consultation, $r_{p b}(59)=-0.39$, $p<0.001$, but no significant relationship with telepresence was found.

A six stage hierarchical multiple regression was conducted with how comfortable telepsychotherapists feel with online consultations as the dependent variable. Previous experience was entered at stage one of the regression, the level of perceived telepresence was entered at stage 2 , and years of experiences at stage 3. Type of training, age and gender were entered at stages 4, 5 and 6 respectively. Full results are presented in Table 2. Multiple linear regressions looking for predictors of how comfortable the therapist felt with telepsychotherapy in the reason why he/ she chose to start using online consultations and looking for predictors of perceived self-rated quality of telepresence in the method of telepsychotherapy were not significant with $F(6,66)=0.54, p=0.78$ and $F(4,55)=1.77$, $p=0.15$ respectively.
Table 2 Hierarchal multiple regression looking for predictors of how comfortable one feels with online consultations

\begin{tabular}{|c|c|c|c|c|c|}
\hline Variable & $\beta$ & $t$ & $R$ & $R^{2}$ & $\Delta R^{2}$ \\
\hline $\begin{array}{l}\text { Step } 1 \\
\text { Previous experience using online consultations }\end{array}$ & -0.24 & $-1.97^{*}$ & 0.28 & 0.08 & 0.08 \\
\hline $\begin{array}{l}\text { Step } 2 \\
\text { Previous experience using online consultations } \\
\text { Level of telepresence }\end{array}$ & 0.38 & $3.08 * *$ & 0.46 & 0.21 & 0.13 \\
\hline $\begin{array}{l}\text { Step } 3 \\
\text { Previous experience using online consultations } \\
\text { Level of telepresence } \\
\text { Years of previous experience }\end{array}$ & -0.28 & -1.40 & 0.50 & 0.25 & 0.05 \\
\hline $\begin{array}{l}\text { Step } 4 \\
\text { Previous experience using online consultations } \\
\text { Level of telepresence } \\
\text { Years of previous experience } \\
\text { Received specific training in online consultations }\end{array}$ & -0.26 & -0.21 & 0.51 & 0.26 & 0.00 \\
\hline $\begin{array}{l}\text { Step } 5 \\
\text { Previous experience using online consultations } \\
\text { Level of telepresence } \\
\text { Years of previous experience } \\
\text { Received specific training in online consultations } \\
\text { Age }\end{array}$ & 0.078 & 0.38 & 0.51 & 0.26 & 0.00 \\
\hline $\begin{array}{l}\text { Step } 6 \\
\text { Previous experience using online consultations } \\
\text { Level of telepresence } \\
\text { Years of previous experience } \\
\text { Received specific training in online consultations } \\
\text { Age } \\
\text { Gender }\end{array}$ & -0.25 & -0.19 & 0.51 & 0.26 & 0.00 \\
\hline
\end{tabular}




\section{Discussion}

The COVID-19 lockdown has forced mental health professionals to partially or fully transfer their practices online, with little time for both patients and mental health professionals to adapt to this change. This study set out to explore reasons why some made the move to telepsychotherapy while others did not and further looked for predictors of how comfortable one is with online consultations in a sample of Lebanese mental health professionals.

$82 \%$ of participants reported having made use of online consultations in recent days, in which the majority justified it as wanting to be there for clients who could not attend, in line with the western literature (Matheson et al., 2020), and some considered it a public health necessity. Others reported that telepsychotherapy has been requested by their clients, about a quarter of professionals did not want to lose income, and lastly $21 \%$ reported wanting to stay in touch with how technology is evolving. Only a small number did not start online consultations, reporting concerns about its effectiveness and considered that online consultations did not provide an added value over continuing face to face. Overall, our results indeed highlighted mental health professionals' attempt to accommodate with the fast-changing circumstances despite some reservations.

Individuals with experience in online consultations prior to the COVID-19 pandemic were more likely to use such technology during the pandemic as well. Gender was also significantly related to the use of online consultations but this did not hold up in the regression model. This could be explained by the fact that $82 \%$ of the sample were female, with the majority making use of online consultations, which could have biased the results. Other factors such as age, professional experience, and training were not significantly related to current use of online consultations. In line with this, mental health professionals' rating of how comfortable they felt with online consultations was significantly moderately correlated with their use of telepsychotherapy prior to COVID-19 and their perceived level of telepresence (online presence) in recent consultations; however, the rating was not significantly associated with having received specific training in online consultations.

The effects of these variables remained when integrated in a regression model as predictors of how comfortable one felt with online consultations. Results of the hierarchal multiple regression highlight that having previous experience with online consultations and level of perceived telepresence explain $13.2 \%$ of the variance in how comfortable the mental health professional feels with online consultations. Adding years of experience, having specific training in online consultations, age and gender did not significantly improve the model. This strengthens previous findings relating to the crucial role played by experience in the delivery of telepsychotherapy and one's confidence regarding the use of this therapy method. Our findings are in line with studies suggesting that the more experience one has with online consultations, the more confident they feel concerning overcoming the challenges of telepsychotherapy, despite still preferring face-to-face meetings (Connolly et al., 2020; Lindsay et al., 2017).

Given that the move to telepsychotherapy was somewhat forced upon mental health professionals due to the COVID-19 circumstances, we were interested in investigating whether the reasons that led mental health professionals to engage in online consultations affected how comfortable they felt with this new practice. However, the regression model was not significant, revealing that none of the reasons leading mental health professionals to make use of online consultations significantly affected their perceived level of self-confidence with the use of this therapy method. This is somewhat surprising but could relate to findings that mental healthcare providers' attitudes towards the use of telepsychotherapy and their experience with it might be influenced by how comfortable one feels concerning telepsychotherapy, rather than by the reason why they decided to use it (Connolly et al., 2020; Lindsay et al., 2017). Over the course of the pandemic, the main reason for the switch to telepsychotherapy seems to have been to remain available for their patients. It can also be argued that mental health professionals would not have taken on telepsychotherapy if they did not have some confidence in their ability to provide it, even if requested by their clients. In fact, participants in our sample scored relatively high on both questions relating to perceived telepresence and how comfortable they felt with the use of online consultations. Future research could focus on exploring these predictors in telepsychotherapists who scored lower on these scales. This result could also be interpreted through a cultural lens, in which Lebanon is described as a collectivistic culture where the group's harmony is prioritized, affecting social attributes and roles (Tohme et al., 2020). In other words, helping others (or any other reason that might have led the healthcare provider to use online consultations) is seen as a duty and separate from one's rating of their confidence at work.

Based on these findings, we suggest the following recommendations for moving forward with telepsychology procedures as part of standard clinical practice. First, promote the therapeutic skills necessary to enhance proficiency and telepresence (perceived online presence) through workshops, online trainings and intervision groups. Future studies could focus specifically on looking for predictors of the mental health professional's rating of online presence and feeling connected to their patients online. This could shed more light the relationship 
between telepresence and level of comfort with online consultations. Second, raise awareness regarding the effectiveness of telepsychotherapy as this may constitute a barrier for professionals as well as clients seeking psychological support. Third, governing bodies and mental health bodies in Lebanon should set a clear list of guidelines to be adhered to in terms of security, confidentiality, and others, ensuring that all mental health professionals making use of online consultations abide by them. However, there also needs to be room for flexibility as online consultations are not suitable for every client or context, like those at risk of suicide or harming others, or younger children with limited verbal and writing skills (Van Daele et al., 2020).

Despite this study being the first to examine the use of teletherapy in Lebanon during the COVID-19 pandemic, it is important to interpret these results in light of some limitations. First, the current study made use of a small convenience sample, and analyses were hampered by small sample sizes in subgroups. Another limitation is that the percentage of professionals transferring their work online could be an overestimation. Indeed, given the pandemic lockdown restrictions, the self-report survey was only circulated online. This could have biased the responses and sample recruited, for it can be argued that those having more ease or familiarity with using social media platforms were more likely to have already started using online consultations.

In sum, our findings support a preliminary adaptation of Lebanese mental health professionals to the circumstances imposed by the COVID-19 lockdown. Results highlight the crucial role of previous experience using online consultations in affecting the use of telepsychotherapy during the COVID-19 enforced lockdown. Professionals' previous use of telepsychotherapy was also found to significantly affect their ratings of how comfortable they felt with online consultations and their rated levels of telepresence. We recommend more awareness about recent developments regarding telepsychotherapy, especially since studies have found that, for some psychological disorders, it could increase treatment adherence (Poletti et al., 2020). One way to do so would be to set up studies comparing the effectiveness of face-to-face traditional therapy and telepsychotherapy through a range of therapeutic approaches, and further exploring whether culture plays a role by contrasting studies in Lebanon with previous findings from the western world. It would be of interest to organize focus groups delving into mental health professionals' views and opinions regarding telepsychotherapy beyond the COVID-19 restrictions, in order to uncover whether this method of therapy delivery might persist in Lebanon. We hope to provide further insight as well as culture-specific and population-specific recommendations in a follow-up study based on the qualitative data analysis of mental healthcare professionals' main concerns about telepsychotherapy.

\section{References}

Ball, C. J., McLaren, P. M., Summerfield, A. B., Lipsedge, M. S., \& Watson, J. P. (1995). A comparison of communication modes in adult psychiatry. Journal of Telemedicine and Telecare, 1(1), 22-26. https://doi.org/10.1177/1357633X9500100105

Connolly, S. L., Miller, C. J., Lindsay, J. A., \& Bauer, M. S. (2020). A systematic review of providers' attitudes toward telemental health via videoconferencing. Clinical Psychology: Science and Practice, 12, e12311. https://doi.org/10.1111/cpsp.12311

De Witte, N. A., Carlbring, P., Etzelmueller, A., Nordgreen, T., Karekla, M., Haddouk, L., Belmont, A., Øverland, S., AbiHabib, R., Bernaerts, S., Brugnera, A., Duque, A., Ebert, D. D., Eimontas, J., Kassianos, A. P., Salgado, J., Schwerdtfeger, A., Tohme, P., Van Assche, E., \& Van Daele, T. (2020). Online consultations in mental healthcare during the Covid-19 outbreak: An International survey study on uptake and experiences. JMIR Preprints. https://doi.org/10.2196/preprints.26541

Glueckauf, R. L., Maheu, M. M., Drude, K. P., Wells, B. A., Wang, Y., Gustafson, D. J., \& Nelson, E. L. (2018). Survey of psychologists' telebehavioral health practices: Technology use, ethical issues, and training needs. Professional Psychology: Research and Practice, 49(3), 205-219. https://doi.org/10.1037/pro00 00188

Haddouk, L. (2015). Presence in telepsychotherapy. Towards a videointerview framework. International Journal of Emergency Mental Health, 17, 712-713. https://doi.org/10.4172/1522-4821.1000296

Haddouk, L., Bouchard, S., Brivio, E., Galimberti, C., \& Trognon, A. (2018). Assessing presence in videoconference telepsychotherapies: A complementary qualitative study on breaks in telepresenceandintersubjectivity co-construction processes. Annual Review of Cybertherapy and Telemedicine, 16, 118-123. https:// doi.org/10.1037/1528-3542.6.3.383

Hadjistavropoulos, H. D., Pugh, N. E., Hesser, H., \& Andersson, G. (2017). Therapeutic alliance in internet-delivered cognitive behaviour therapy for depression or generalized anxiety. Clinical Psychology \& Psychotherapy, 24(2), 451-461. https://doi. org/10.1002/cpp.2014

Jenkins-Guarnieri, M. A., Pruitt, L. D., Luxton, D. D., \& Johnson, K. (2015). Patient perceptions of telemental health: Systematic review of direct comparisons to in-person psychotherapeutic treatments. Telemedicine and e-Health, 21(8), 652-660. https:// doi.org/10.1089/tmj.2014.0165

Kocsis, B. J., \& Yellowlees, P. (2018). Telepsychotherapy and the therapeutic relationship: Principles, advantages, and case examples. Telemedicine and e-Health, 24(5), 329-334. https://doi. org/10.1089/tmj.2017.0088

Lindsay, J. A., Hudson, S., Martin, L., Hogan, J. B., Nessim, M., Graves, L., \& White, D. (2017). Implementing video to home to increase access to evidence-based psychotherapy for rural veterans. Journal of Technology in Behavioral Science, 2(3-4), 140-148. https://doi.org/10.1007/s41347-017-0032-4

Matheson, B., Bohon, C., \& Lock, J. (2020). Family-based Treatment via videoconference: Clinical recommendations for treatment providers during COVID-19 and beyond. Preprint. https://doi. org/10.31234/osf.io/b3hcs

O'Malley, C., Langton, S., Anderson, A., Doherty-Sneddon, G., \& Bruce, V. (1996). Comparison of face-to-face and video-mediated interaction. Interacting with Computers, 8(2), 177-192. https://doi.org/10.1016/0953-5438(96)01027-2

Poletti, B., Tagini, S., Brugnera, A., Parolin, L., Pievani, L., Ferrucci, R., \& Silani, V. (2020). Telepsychotherapy: A leaflet for psychotherapists in the age of COVID-19. A review of the evidence. Counselling Psychology Quarterly, 12, 1-16. https://doi.org/10. 1080/09515070.2020.1769557 
Ryu, S. (2010). History of telemedicine: Evolution, context, and transformation. Healthcare Informatics Research, 16(1), 65-66. https:// doi.org/10.4258/hir.2010.16.1.65

Shore, J. H., Yellowlees, P., Caudill, R., Johnston, B., Turvey, C., Mishkind, M., Krupinski, E., Myers, K., Shore, P., Kaftarian, E., \& Hilty, D. (2018). Best practices in videoconferencing-based telemental health April 2018. Telemedicine and E-Health, 24(11), 827-832. https://doi.org/10.1089/tmj.2018.0237

Stewart, R. W., Orengo-Aguayo, R., Young, J., Wallace, M. M., Cohen, J. A., Mannarino, A. P., \& de Arellano, M. A. (2020). Feasibility and effectiveness of a telehealth service delivery model for treating childhood posttraumatic stress: A community-based, open pilot trial of trauma-focused cognitive-behavioral therapy. Journal of Psychotherapy Integration, 30(2), 274-289. https://doi.org/ 10.1037/int0000225

Tohme, P., Grey, I., \& Abi-Habib, R. (2020). The Mental States Task (MST): Correlates and new perspectives on mentalizing in a Lebanese student sample. Journal of Personality Assessment. https:// doi.org/10.1080/00223891.2020.1769114

Van Daele, T., Karekla, M., Kassianos, A. P., Compare, A., Haddouk, L., Salgado, J., Ebert, D. D., Trebbi, G., (on behalf of the EFPA
Project Group on eHealth), Bernaerts, S., Van Assche, E., \& De Witte, N. A. J. (2020). Recommendations for policy and practice of telepsychotherapy and e-mental health in Europe and beyond. Journal of Psychotherapy Integration, 30(2), 160-173. https://doi. org/10.1037/int0000218

Venkatesh, V., Morris, M. G., Davis, G. B., \& Davis, F. D. (2003). User acceptance of information technology: Toward a unified view. MIS Quarterly, 27, 425-478

Wade, S. L., Gies, L. M., Fisher, A. P., Moscato, E. L., Adlam, A. R., Bardoni, A., ... Williams, T. (2020). Telepsychotherapy with children and families: Lessons gleaned from two decades of translational research. Journal of Psychotherapy Integration, 30(2), 332-347. https://doi.org/10.1037/int0000215

Publisher's Note Springer Nature remains neutral with regard to jurisdictional claims in published maps and institutional affiliations. 